\title{
Clinical and Radiological Outcomes of Segmental Spinal Fusion in Transforaminal Lumbar Interbody Fusion with Spinous Process Tricortical Autograft
}

\author{
Nattawat Witoon, Teera Tangviriyapaiboon \\ Department of Neurosurgery, Prasat Neurological Institute, Bangkok, Thailand
}

\section{Study Design: A retrospective study.}

Purpose: To investigate clinical and radiological outcomes when using spinous process as a tricortical autograft for segmental spinal fusion in transforaminal lumbar interbody fusion (TLIF).

Overview of Literature: Interbody spinal fusion is one of the important procedures in spinal surgery. Many types of autografts are harvested at the expense of complications. Clinical and radiographic results of patients who underwent TLIF with intraoperative harvested spinous process autograft in Prasat Neurological Institue, Bangkok, Thailand, were assessed as new technical innovation. Methods: Between October 2005 to July 2009, 30 cases of patients who underwent TLIF with spinous process tricortical autograft were included. Clinical evaluations were assessed by visual analog scales (VAS) and Prolo functional and economic scores at the preoperation and postoperation and at 2 years postoperation. Static and dynamic plain radiograph of lumbar spine were reviewed for achievement of fusion.

Results: Initial successful fusion time in lumbar interbody fusion with spinous process tricortical autograft was 4.72 months (range, 3.8-6.1 months) postoperation and 100\% fusion rate was reported at 2 years. Our initial successful fusion time in lumbar interbody fusion was compared to the other types of grafts in previous literatures.

Conclusions: The use of intraoperative harvested spinous process tricortical autograft has overcome many disadvantages of harvesting autograft with better initial successful fusion time (4.72 months). VAS and Prolo scores showed some improvement in the outcomes between the preoperative and postoperative periods.

Keywords: Transpedicular screw fixation; Transforaminal lumbar interbody fusion; Spinous process autograft; Tricortical autograft

\section{Introduction}

Segmental spinal fusion is one of the common procedures to perform spinal surgery. This concept of fusion was first introduced by Albee and Hibbs in 1911 as the treatment of Pott disease with posterior spinal fusion by autogenous bone graft [1,2]. In 1933, lumbar interbody fusion was first performed by Burns for the treatment of traumatic L5-S1 spondylolisthesis via the transabdominal approach. The rate of fusion was unsatisfied. Not until the development of instrumentation system in mid-last century, which improved the fusion rate to more than $90 \%[2-6]$.

Many donor sites are selected and harvested for auto-

Received Nov 29, 2012; Revised Jan 25, 2013; Accepted Jan 31, 2013

Corresponding author: Nattawat Witoon

Department of Neurosurgery, Prasat Neurological Institute,

312 Rajvithi Rd., Rajthewee District, Bangkok 10400, Thailand

Tel: +66-2-354-7072-9, Fax: +66-2-354-7080, E-mail: tonnattawat_mhs@hotmail.com 
graft in spinal segmental fusion, such as iliac crest, fibula, rib, and etc. Many advantages of autografts were described in recent literatures, such as containing of natural combination substances and cells to promote fusion, no additional risk of disease transmission and cost effectiveness. Some disadvantages had drawn back the utilization of autograft, including harvest site morbidity, limit availability, additional incision, increased operative time, and etc. $[2,4,7]$.

In this study, the senior author (T.T.) has introduced a new option for grafting in transforaminal lumbar interbody fusion (TLIF), consideration on the bone that was obtained from laminectomy as a useful source of autograft. Each spinous process is cut in whole pieces (Fig. 1) and provides as a tricortical autograft as Fig. 2, the spinous process autograft is prepared to disc space via the transforaminal route as TLIF cage. We studied regarding the timing and rate of fusion with spinous process of tricortical autograft in TLIF of up to 2 years after the operation, compared with fusion rate and time of those were reported in previous known literatures. The patient clinicals, visual analog scales (VAS) and Prolo functional and economic scales were reviewed, preoperative, at the time of initial successful fusion and 2 years postoperation.
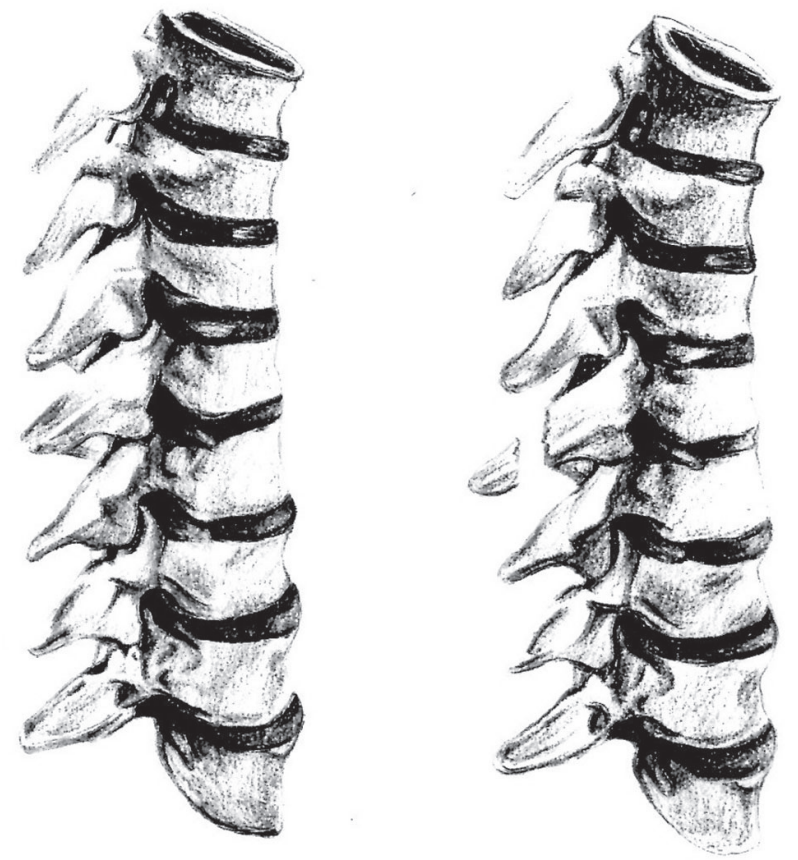

Fig. 1. Courtesy of Netter diagram. The picture drawn by Siriporn Kaowiwattanakul.
Table 1. Basic characteristics of patients

\begin{tabular}{|c|c|}
\hline Characteristic & $\mathrm{n}(\%)$ \\
\hline Gender & 30 \\
\hline Male & $8(26)$ \\
\hline Female & $22(73)$ \\
\hline \multicolumn{2}{|l|}{ Age in years on admission date } \\
\hline $40-49$ & $5(16)$ \\
\hline $50-59$ & $14(46)$ \\
\hline $60-69$ & $9(30)$ \\
\hline $70-79$ & $2(6)$ \\
\hline \multicolumn{2}{|l|}{ Chief complaints } \\
\hline Radicular pain & $25(83)$ \\
\hline Motor weakness & $3(10)$ \\
\hline Sensory impairment & $2(6)$ \\
\hline \multicolumn{2}{|l|}{ Frequency of diagnosis } \\
\hline Spondylolisthesis & $27(56)$ \\
\hline Spinal canal stenosis & $15(31)$ \\
\hline Degenerative disc disease & $6(12)$ \\
\hline \multicolumn{2}{|l|}{ Level of TPS insertion } \\
\hline L2-3 & $5(9)$ \\
\hline L3-4 & $13(23)$ \\
\hline$\llcorner 4-5$ & $26(46)$ \\
\hline L5-S1 & $12(21)$ \\
\hline Level of fusion & 50 \\
\hline L2-3 & $5(10)$ \\
\hline L3-4 & $12(24)$ \\
\hline L4-5 & $24(48)$ \\
\hline L5-S1 & $9(18)$ \\
\hline
\end{tabular}

TPS, transpedicular screws.

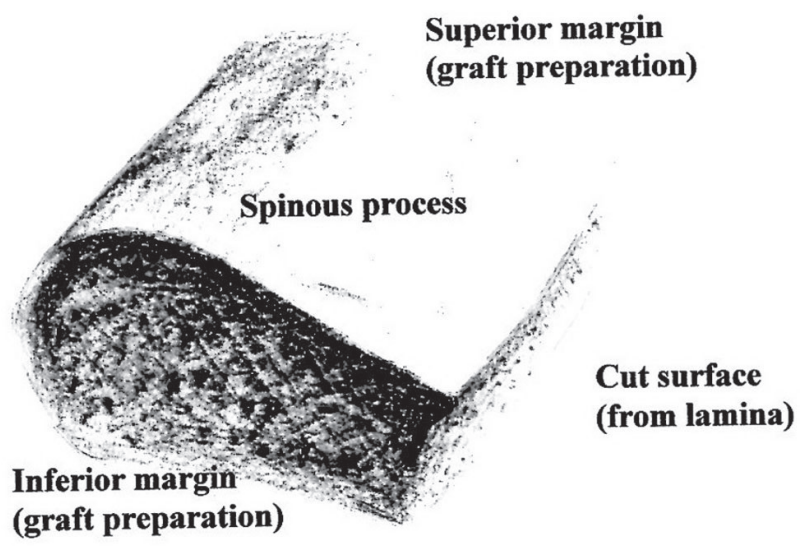

Fig. 2. Courtesy of BioCleanse tissue sterilization process. The picture drawn by Siriporn Kaowiwattanakul. 


\section{Materials and Methods}

\section{Patient characteristics and patient selections}

Retrospective reviews of medical records and radiographs were conducted in 30 patients who were admitted and underwent transpedicular screws insertion with TLIF at Prasat Neurological Institute, Bangkok, Thailand, between October 2005 and July 2009 (Table 1).

Regardless of the diagnosis, fifty levels of spinous process tricortical autograft interbody fusion were collected in this study. Of the 30 patients, 8 were men $(26 \%)$ and 22 were women $(73 \%)$. The patients mean age was 58.03 years (range, 43-76 years). Clinical presentations were radicular pain in 25 patients (83\%), motor weakness in 3 patients (10\%), and sensory impairment in 2 patients (6.6\%). The diagnosis can be classified into spondylolisthesis, degenerative disc disease and spinal canal stenosis. They were fifty vertebral levels involved in the interbody fusion in this study, categorized into L2-L3 5 levels (10\%), L3-L4 12 levels (24\%), L4-L5 24 levels (48\%), and L5-S1 9 levels (18\%).

\section{Clinical assessments}

Retrospective reviews of hospital charts were conducted to access age, gender, preoperative symptomatology, clinical presentations, VAS, diagnosis, operative record reviewed for level of transpedicular screw insertion, interbody fusion and date of operation. Postoperative clinicals were evaluated by Prolo functional and economic scales at the time of initial successful fusion and at 2 years postoperation.

\section{Radiographic assessments}

All available postoperative radiological studies, plain film of lumbar spine anteroposterior, lateral and flexion-extension views were reviewed by the authors for evidence of segmental spinal fusion. Each case, radiographical studies were randomize-reviewed, unaware of clinical results. The successful fusion is defined as [4,6,8,9]: 1) Absence of halo around the screws; 2) Presence of bilateral continuous trabecular bone bridge between the fused segments on the anteroposterior plain film; 3) Lack of motion on the flexion-extension film (Fig. 3).
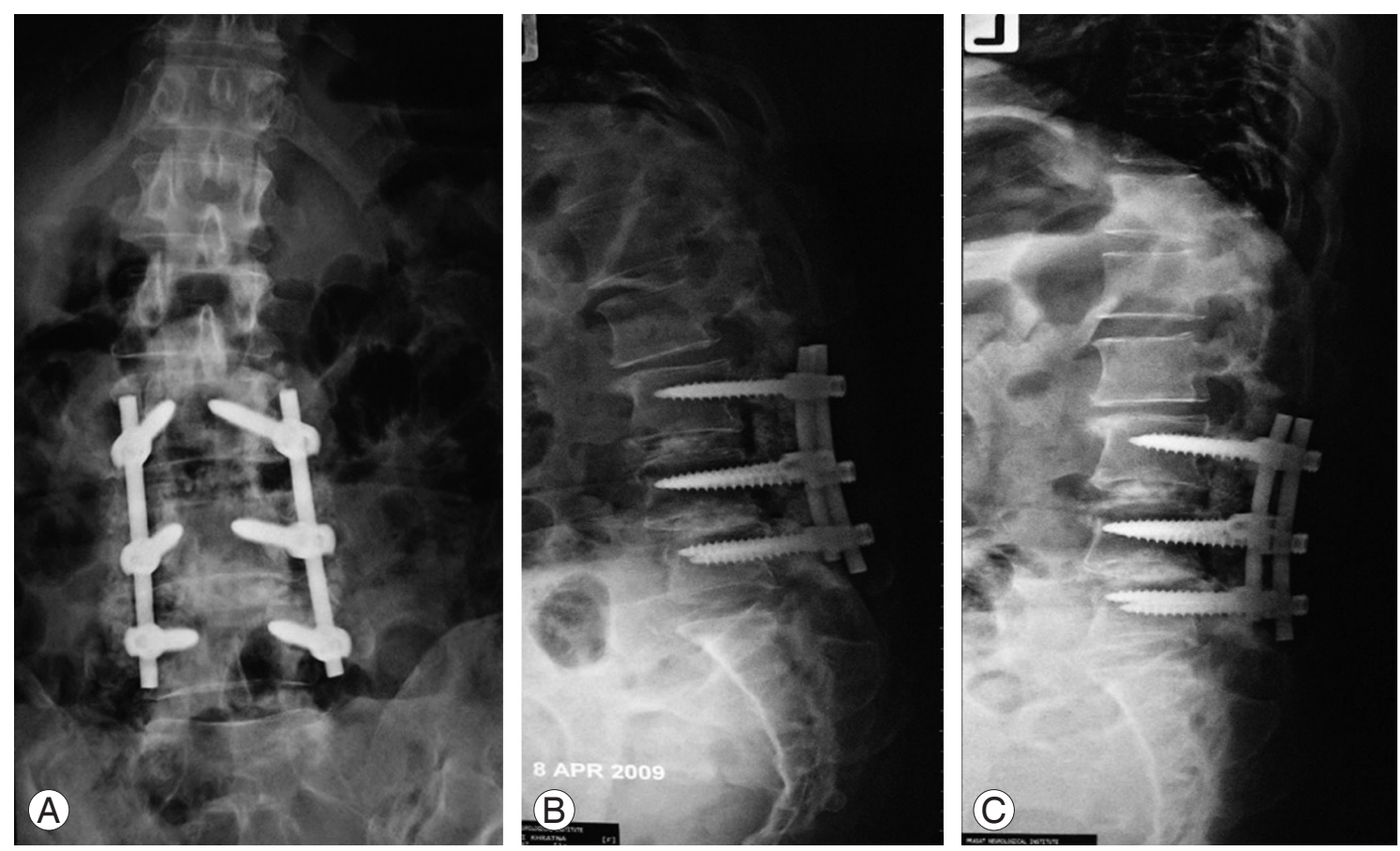

Fig. 3. (A) A 49-year-old female patient, 6 months after transpedicular screw insertions and transforaminal lumbar interbody fusion at L3-L4-L5 showed bilateral trabecular bone bridge at L4-L5 space on anteroposterior plain film. (B, C) On Lateral view, there were no halo around screws and lack of motion of fused segment on flexion-extension film. 
Table 2. Average fusion time

\begin{tabular}{lcc} 
Level of fusion & No. (\%) & $\begin{array}{c}\text { Average fusion time } \\
\text { (mo) }\end{array}$ \\
\hline L2-3 & $5(10)$ & 3.8 \\
L3-4 & $12(24)$ & 4.5 \\
L4-5 & $24(48)$ & 4.5 \\
L5-S1 & $9(18)$ & 6.1 \\
Overall average & - & 4.72 \\
\hline
\end{tabular}

Table 3. Clinical evaluation scores

\begin{tabular}{lcc} 
& $\begin{array}{c}\text { Average visual } \\
\text { analog scale }\end{array}$ & $\begin{array}{c}\text { Prolo functional } \\
\text { and economic score }\end{array}$ \\
Preoperation & 8.72 & - \\
At time of fusion & 4.68 & 7.9 \\
2 Years postoperation & 3.92 & 8.5 \\
\hline
\end{tabular}

\section{Surgical technique}

The patient is positioned prone on a radiolucent table. Appropriate spinal level is identified by a C-arm fluoroscopy. Midline skin incision is made. Paraspinal muscles are bluntly dissected. Spinous processes, lamina and bilateral facet joints are identified. After transpidicular screws are inserted, supraspinatous and interspinatous ligaments of identified levels are incised. The entire spinous process is cut in whole pieces (Fig. 1), using a bone cutter, harvested for interbody fusion autograft. Total laminectomy is done and unilateral facetectomy is done on the side ipsilateral to the lower extremity symptom. The exiting nerve root is identified and protected with a small discector. Aggressive removal of the disc tissue is performed on the side of facetectomy. Interbody fusion is done, using spinous process tricortical autograft placed fit into the disc space. The posterior instrumentation is then tightened. Posterior lateral fusion is performed before closing suture of the paraspinal muscle and skin are made.

\section{Results}

We included 30 cases of patients who underwent transpedicular screws insertion and TLIF with spinous process tricortical autograft in Prasat Neurological Institute, Bangkok and Thailand, between October 2005 and July 2009. These groups of patients underwent 50 levels of lumbar interbody fusion.

The average successful fusion time is 4.72 months (range, 2-11 months), postoperation. The average initial fusion times are classified by levels; L2-L3 3.8 months, L3-L4 4.5 months, L4-L5 4.5 months, and L5-S1 6.1 months (Table 2). At 2 years postoperation, all cases fulfilled the criteria of successful fusion (rate of successful fusion is $100 \%$ ). However, instrumentation failures were identified in 3 cases. Case no. 4 , broken of screws at the left L5 17 months postoperation; case no. 7, broken of screws at the right L5 6 months postoperation, the last case, case no. 11, and loosening of screws at the right S1 17 months postoperation. Without instability, only the last case required reoperation for screw revision due to an increase of radicular pain from malposition of screw.

VAS was reviewed in 25 cases (83\%). Of these cases, 6 cases $(24 \%)$ were presented with right leg radicular pain, 10 cases $(40 \%)$ presented with left leg pain and 9 cases (36\%) with leg pain presented on both sides. The average preoperative VAS is 8.72 (range, 7-10). The average VAS at the time of successful fusion and at 2 years postoperation are 4.68 (range, 0-6) and 3.92 (range, 0-5) (Table 3 ). The 3 patients (10\%) were case nos. 7,14 , and 16 with motor weakness on preoperative clinical evaluation. Only one case (case no. 14) reported improvement of motor weakness from no movement of Extensor hallicis longus (EHL) on preoperative evaluation to grade-2 motor power on postoperative examination. Further, 2 patients $(6.7 \%)$ (case nos. 11,18 ) were presented with profound sensory loss of lower extremities. Symptoms were unchanged on follow up clinical evaluation.

Prolo functional and economic scales (Table 4) were observed in this study at the time of initial successful fusion and at 2 years postoperation. The results of Prolo scale scores at the time of initial successful fusion are excellent outcomes (score of 9-10) for 11 cases (36.7\%), good outcomes (score of 7-8) for 14 cases (46.7\%), fair outcomes (score of 5-6) for 4 cases (13\%) and 1 case $(0.3 \%)$ reported for poor outcome (score of $\leq 4)$. The Prolo scale scores were also recorded at 2 years postoperation, with 16 cases (53\%) of excellent outcomes, 12 cases (40\%) of good outcomes, 2 cases $(6.7 \%)$ of fair outcomes and no case was reported as poor outcome (Table 5). The Prolo scale scores are compared between the time of successful fusion and 2 years postoperation, with which the results are variable. Stable of scores in 12 cases (40\%), improvement in score in 16 cases (53\%) and 2 cases (6.7\%) 
Table 4. Summary of the Prolo functional and economic scales

\begin{tabular}{|c|c|} 
Functional status & \multicolumn{1}{c}{ Criteria } \\
\hline 1 & Total incapacity \\
\hline 2 & Mild to moderate level of low back pain and/or sciatica pain \\
\hline 3 & Low level of pain and able to do everything except sports \\
\hline 4 & No pain, but has had $>1$ recurrence of low back pain or sciatica \\
\hline 5 & Complete recovery without recurrence pain, no activity restriction \\
\hline Economic status & Complete disability \\
\hline 1 & No gain occupation, but can do house work or some retirement activities \\
\hline 3 & Able to work, but not at previous occupation \\
\hline 5 & Able to work at previous occupation, but with restrictions or limited status \\
\hline
\end{tabular}

w/o, Please define this abbreviation.

Table 5. Comparison number of cases of Prolo functional and economic scores between at time of initial fusion and 2 years postoperation

\begin{tabular}{|c|c|c|}
\hline Outcome & At time of initial fusion & 2 Years post operation \\
\hline Excellent (9-10) & 11 & 16 \\
\hline Good (7-8) & 14 & 12 \\
\hline Fair (5-6) & 4 & 2 \\
\hline Poor $(\leq 4)$ & 1 & - \\
\hline Average scores & 7.9 & 8.5 \\
\hline
\end{tabular}

Table 6. Comparison of outcome of VAS and Prolo functional and economic scores between at time of initial fusion and 2 years postoperation

\begin{tabular}{lcc} 
Scores & $\begin{array}{c}\text { Visual analog } \\
\text { scale (VAS) }\end{array}$ & $\begin{array}{c}\text { Prolo functional and } \\
\text { economic scores }\end{array}$ \\
\hline Stable & 7 & 12 \\
Improved scores & 16 & 16 \\
Worsening scores & 2 & 2 \\
\hline Total & 25 & 30 \\
\hline
\end{tabular}

reported worsening of the score (Table 6).

\section{Disscussion}

In spinal surgery, fusion is one of the most important procedures and concepts of treatment. In the early of twentieth century, spinal fusion was achieved by placement of tibial graft between spinous processes for treatment of Pott disease. The idea of creating a rigid union between vertebral segments to correct spinal column instability was the fundamental of further treatment and concept in spinal fusion over the next several decades, even their high rate of pseudoarthrosis. Until 1933, Burns introduced a new fusion technique through the anterior approach and interbody fusion, in which the autograft was placed into the interbody space [2]. Rate of successful fusion increased to more than $50 \%$. Not until the mid of the last century, many spinal instrumentation systems were introduced. Some series had reported successful fusion rate of interbody fusion combined with instrumentation of more than $90 \%$ [2-6].

Many types of grafts are used for the interbody fusion. Autograft is an ideal and gold standard graft compared to other graft materials because it contains the natural combination of osteogenic, osteoconductive and osteoconductive properties. Many types of autografts are considered e.g., iliac crest, morcellized spinal bone, structural grafts (fibula, humerus, femur), and etc. $[2,4,7]$.

In the recent studies, the rate of successful interbody fusion with instrumentations is variable due to the type of grafts. Fibula and morcellized autograft, and successful 
Table 7. Time of initial fusion by types of graft

\begin{tabular}{lc} 
Types of graft & $\begin{array}{c}\text { Time of initial } \\
\text { fusion (mo) }\end{array}$ \\
\hline Autugraft plus rhBMP2 & 3 \\
\hline Tricortical spinous process autograft & $4.72^{\text {a) }}$ \\
\hline Titanium cage & 6 \\
Titanium cage packed with autograft & 7.4 \\
Ray threaded fusion cage & 12 \\
\hline
\end{tabular}

rhBMP2, recombinant human bone morphogenetic protein 2 .

a) The result of our study.

interboby fusion were presented at 11 months postoperation. Titanium cage packed with autologous bone graft, initial segmental fusion took place at 6 to 7.4 months. The earliest successful fusion, 2 months, was presented with autograft plus osteoinductive substances, such as recombinant human bone morphogenetic protein 2 (rhBMP2). Interbody fusion with Ray-threaded fusion cage presented the longest duration of 12 months for initial fusion took place on plain film (Table 7).

The senior author of our study (T.T.) has introduced the new option for bone graft for interbody fusion. We use spinous process from laminectomy as tricortical bone graft, which inserted to the intervertebral space via transforaminal route.

This study, we evaluated the initial successful of fusion by plain radiographs, despite accuracy is only two-third of cases when compared with the gold standard fusion assessment, "direct surgical exploration." The accuracy of plain radiographs can be increased with dynamic (flexion-extension) radiographs, with positive predictive value of $70 \%$ and negative predictive value of $86 \%$ compared to positive predictive value of $76 \%$ and negative predictive value of $54 \%$ on static film. The term "successful fusion" was indicated when all three criteria as mentioned earlier in this study are met.

In our study, we found that the average time of successful interbody fusion is 4.72 months. There are 3 levels that met the successful fusion criteria, earliest at 2 months postoperation and one level that lastest fulfill fusion criteria at 11 months. The spinous process tricortical autograft revealed initial successful fusion time better than other type of autograft and artificial graft as we mentioned earlier in this study, except only in the autograft plus rhBMP2 group. Of the harvesting processes, graft preparation is one of the important procedures.
After spinous process was cut in whole piece, using bone ronguer to knit the superior and inferior surfaces of the spinous process, it exposed the cancellous surfaces (Fig. 2) and trimmed the graft for proper fitting into interlumbar space. We counted three on cortical surfaces as tricortical graft. This provided good structural support for the graft to maintain shape of the graft and disc space height under compression. Furthermore, the spinous process tricortical autograft also provided osteoconductive, osteoinductive and osteogenesis properties as the fresh graft that help in promoting fusion.

The intraoperative graft harvesting provides opportunity to overcome the disadvantages of autograft harvesting, such as no need for additional incision, decrease operative time and blood loss. However, we still concern about the adequacy and quality of spinous process autograft. In some cases, the spinous processes are small, abnormally contour or osteoporotic bone in nature, which can cause pseudoarthrosis.

Despite the 3 cases that were identified as instrumentation failures, which occurred at 6 and 17 months postoperation. On 2 years postoperative follow up, the rate of successful fusion is $100 \%$. This is due to timing of the initial successful fusion that took place earlier at 4.72 months before the failure of instrumentation.

The VAS were reviewed in 25 patients who were presented with lower extremities pain. The preoperative VAS is 8.72 . We recorded VAS at the time of initial fusion, and the average VAS is 4.68 , corresponding to immobility of spinal segment, which pointed to the initial successful fusion. As we compared the preoperative VAS at the time of fusion and postoperative VAS, which was $100 \%$ improved, implied that clinical recovery of patients may be due to fixation of mobility spinal segment and virtue of successful fusion at those time. However, the VAS was followed up to 2 years, which was compared to VAS at the time of the initial successful fusion. The results are variable. We pay attention to 2 cases of worsening VAS. These patients complained about the newly developed radicular pain on contralateral sides, despite good position of the instruments and fusion, which might be due to recurrent spinal canal stenosis, epidural scar or adjacent level syndrome.

The Prolo functional and economic scales were observed in this study. We reported excellent and good outcomes scores as the "response to treatment" in 25 cases $(83 \%)$ at the time of initial successful fusion and 26 cases 
$(86.7 \%)$ at 2 years postoperation. The response in the treatment group as compared, results are stable and no report of poor outcome score at 2 years postoperation (we found one case of poor outcome, score of 4 , presented with motor grade 0 of the left tibialis anterior and extensor hallicis longus and no motor improvement on followup, but Prolo score increased to 6 . This related to good fixation and fusion on course of follow up at 2 years.

In this study, we found that pain had the most response to surgical treatment as the results of VAS, not with motor weakness and sensory loss, which showed no clinical recovery on a follow-up period, as mentioned in other literatures with good recovery for radicular pain. For recovery of motor weakness and sensory loss might depend on the preoperative duration of symptoms and grading of motor weakness or sensory loss $[6,7,10,11]$.

\section{Conclusions}

Interbody fusion with intraoperative harvested spinous process autograft revealed better initial successful fusion time than other types of grafts, except only when rhBMP2 was available. This might be the method that can solve with harvested site complications. However, quality and contour of spinous process bone must be carefully considered as an important cause of peudarthrosis. We also reported $100 \%$ fusion rate at 2 years postoperation with this fusion technique. VAS and Prolo functional and economic scores were observed and reported as satisfied outcomes as we recorded the results at the time of initial fusion and 2 years postoperation. This result gets along with the fusion time, which as a confirmation for successful fusion condition.

\section{Conflict of Interest}

No potential conflict of interest relevant to this article was reported.

\section{References}

1. La Rosa G, Cacciola F, Conti A, et al. Posterior fusion compared with posterior interbody fusion in segmental spinal fixation for adult spondylolisthesis.
Neurosurg Focus 2001;10:E9.

2. Chang S, Crawford NR, Sonntag VK, Dickman CA. Basic principles of spinal internal fixation. In: Winn HR, editor. Youmans neurological surgery. 6th ed. Philadelphia, PA: Elsevier Saunders; 2011. p.2979-91.

3. Holly LT, Schwender JD, Rouben DP, Foley KT. Minimally invasive transforaminal lumbar interbody fusion: indications, technique, and complications. Neurosurg Focus 2006;20:E6.

4. Tangviriyapaiboon T. Mini-open transforaminal lumbar interbody fusion. J Med Assoc Thai 2008;91:1368-76.

5. Deutsch H, Musacchio MJ Jr. Minimally invasive transforaminal lumbar interbody fusion with unilateral pedicle screw fixation. Neurosurg Focus 2006;20:E10.

6. Klara PM, Freidank SA, Rezaiamiri S. Comparison of lumbar interbody fusion techniques using ray threaded fusion cages and pedicle screw fixation systems. Neurosurg Q 2003;13:20-9.

7. Beringer WF, Mobasser JP. Unilateral pedicle screw instrumentation for minimally invasive transforaminal lumbar interbody fusion. Neurosurg Focus 2006;20:E4.

8. Houten JK, Post NH, Dryer JW, Errico TJ. Clinical and radiographically/neuroimaging documented outcome in transforaminal lumbar interbody fusion. Neurosurg Focus 2006;20:E8.

9. Sheehan JP, Helm GA, Sheehan JM, Jane JA Sr. Stress fracture of the pedicle after extensive decompression and contralateral posterior fusion for lumbar stenosis: report of three cases. Neurosurg Focus 2002;13:E9.

10. Tuttle J, Shakir A, Choudhri HF. Paramedian approach for transforaminal lumbar interbody fusion with unilateral pedicle screw fixation. Technical note and preliminary report on 47 cases. Neurosurg Focus 2006;20:E5.

11. Beringer WF, Mobasser JP, Karahalios D, Potts EA. Anterior transvertebral interbody cage with posterior transdiscal pedicle screw instrumentation for highgrade spondylolisthesis: technical note. Neurosurg Focus 2006;20:E7. 